\title{
Genome-wide association study on serum alkaline phosphatase levels in a Chinese population
}

Jun $\mathrm{Li}^{1 \dagger}$, Lixuan Gui ${ }^{1 \dagger}$, Chen Wu² , Yunfeng He${ }^{1}$, Li Zhou ${ }^{1,5}$, Huan Guo ${ }^{1}$, Jing Yuan ${ }^{1}$, Binyao Yang ${ }^{1}$, Xiayun Dai ${ }^{1}$, Qifei Deng ${ }^{1}$, Suli Huang ${ }^{1}$, Lei Guan' ${ }^{1}$ Die Hu${ }^{1}$, Siyun Deng ${ }^{1}$, Tian Wang ${ }^{1}$, Jiang Zhu ${ }^{3}$, Xinwen Min ${ }^{3}$, Mingjian Lang ${ }^{3}$, Dongfeng $\mathrm{Li}^{3}$, Handong Yang ${ }^{3}$, Frank B Hu${ }^{4}$, Dongxin $\mathrm{Lin}^{2}$, Tangchun $\mathrm{Wu}^{1}$ and Meian $\mathrm{He}^{1 *}$

\begin{abstract}
Background: Serum alkaline phosphatase (ALP) is a complex phenotype influenced by both genetic and environmental factors. Recent Genome-Wide Association Studies (GWAS) have identified several loci affecting ALP levels; however, such studies in Chinese populations are limited. We performed a GWAS analyzing the association between 658,288 autosomal SNPs and serum ALP in 1,461 subjects, and replicated the top SNPs in an additional 8,830 healthy Chinese Han individuals. The interactions between significant locus and environmental factors on serum ALP levels were further investigated.

Results: The association between $A B O$ locus and serum ALP levels was replicated $\left(P=2.50 \times 10^{-21}, 1.12 \times 10^{-56}\right.$ and $2.82 \times 10^{-27}$ for SNP rs8176720, rs651007 and rs7025162 on ABO locus, respectively). SNP rs651007 accounted for $2.15 \%$ of the total variance of serum ALP levels independently of the other 2 SNPS. When comparing our findings with previously published studies, ethnic differences were observed across populations. A significant interaction between $A B O$ rs651007 and overweight and obesity was observed (FDR for interaction was 0.036); for individuals with GG genotype, those with normal weight and those who were overweight or obese have similar serum ALP concentrations; minor allele A of rs651007 remarkably reduced serum ALP levels, but this effect was attenuated in overweight and obese individuals.
\end{abstract}

Conclusions: Our findings indicate that $A B O$ locus is a major determinant for serum ALP levels in Chinese Han population. Overweight and obesity modifies the effect of $A B O$ locus on serum ALP concentrations.

Keywords: Genetic variations, Serum alkaline phosphatase, Heterogeneity, GWAS, Gene-environment interaction

\section{Background}

Alkaline phosphatases (ALP), which is essential in bone mineralization and functions in vascular calcification [1], is a group of hydrolytic isoenzymes with low substrate specificity that catalyze the hydrolysis of organic phosphate esters at an alkaline environment [2]. Four genes encode ALP, including tissue-nonspecific ALP gene located on $1 \mathrm{p} 36.12$, which is expressed in various tissues such as osteoblasts, hepatocytes, kidney and early placenta;

\footnotetext{
* Correspondence: hemeian@hotmail.com

${ }^{+}$Equal contributors

'MOE Key Lab of Environment and Health, School of Public Health, Tongji Medical College, Huazhong University of Science \& Technology, 430030

Wuhan, Hubei, China

Full list of author information is available at the end of the article
}

and three tissue-specific ALP genes located on 2q37, which are expressed in placenta, germ cells, and intestine, respectively [3]. In healthy individuals, serum ALP derives mostly from liver/bone/kidney, partially from intestine especially during postprandial time or in blood type $\mathrm{O}$ or $B$ secretors, and little is contributed from placenta in the third trimester of pregnancy $[4,5]$. The concentration of serum ALP is elevated in pathological conditions like osteoblast activated bone disorders, bile-flow obstructions, tumor metastases, liver diseases, leukemia, hyperthyroidism, infections and obesity, while lower level occurs in anemia and hypothyroidism [2,6]. Current evidence demonstrated that elevated serum ALP levels were associated with adverse outcomes of dialysis patients and increased 
all-cause death rate in myocardial infraction survivors [7-9]. In particular, positive associations between ALP levels and all-cause death rate together with cardiovascular mortality were also observed in the general population [9]. Therefore, investigating the underlying factors that influence serum ALP levels are meaningful for both clinical medicine and public health.

Serum ALP is a complex trait influenced by both polygenic and environmental factors. It is reported that age, gender, smoking, diet, body mass index (BMI), and physical activities could affect serum ALP concentrations [4,10-13]. The additive genetic heritability of serum ALP in mice is approximately 56\% [14]. Recent GWAS have identified several loci, including NBPF3-ALPL, $A B C B 11$, GPLD1, PPP1R3B, TRIB1, ABO, JMJD1C, REEP3, FADS2, PMFBP1, DLG4, FUT2 and ABHD12, associated with serum ALP levels in European ancestry populations, Asian-Indians and Japanese populations [15-17], however, such researches in populations of Chinese origin are limited, and no study investigated gene-environment interactions on serum ALP levels. In the present study, we performed a GWAS in 1,461 subjects and replicated the top SNPs in additional 8,830 healthy Chinese Han individuals. The purposes of the study were to (i) detect genetic determinants for serum ALP in Chinese Han population; (ii) compare the present results with published findings to seek heterogeneity among Chinese and other populations and (iii) investigate the gene-environment interactions on serum ALP levels.

\section{Results}

\section{Characteristics of the study population}

A total of 1,452 subjects $(21.8 \%$ female, $63.05 \pm 8.14$ years old) were included in the discovery set with a mean (SD) serum ALP concentration of 90.02 (26.51) U/L; validation were conducted in 8,830 individuals (58.2\% female, $61.95 \pm 7.83$ years old) whose mean (SD) serum ALP concentration was 90.26 (35.41) U/L. The demographics of the study population together with information on potential influencing factors of serum ALP are summarized in Additional file 1: Table S1.

\section{Association with alkaline phosphatase level}

In GWAS stage, a total of 906,703 SNPs were genotyped among 1,461 subjects in which 38,446 SNPs not mapped on autosomes were excluded. After QC filtering, SNPs with minor allele frequency (MAF) $<0.01$, Hardy-Weinberg Equilibrium $(\mathrm{HWE})<0.0001$, and SNPs call rate $<95 \%$ were excluded. Individuals with a call rate $<95 \%$ were also ruled out for further analysis. Finally, 1,452 subjects with 658,288 autosomal SNPs were retained for association analyses, with an overall call rate of 99.68\%. Quantile - Quantile plot revealed a good match between the distributions of the observed $P$ values and those expected by chance, and a genomic inflation factor of 1.011 suggested that population stratification effects were negligible in our study samples (Additional file 2: Figure S1). The Manhattan plot showed that 2p11.2 (lead SNP rs7594727, $P=3.70 \times 10^{-5}$ ), 2p23.1 (lead SNP rs1383023, $P=2.30 \times 10^{-6}$ ), 9q34.2 (lead SNP rs651007, $P=1.93 \times 10^{-21}$ ) and $17 \mathrm{q} 21.32$ (lead SNP rs7214920, $P=2.55 \times 10^{-6}$ ) were associated with serum ALP at $5.0 \times 10^{-5}$ significance level (Figure 1, Table 1). The regional association plot revealed the SNP-ALP associations and linkage disequilibrium (LD) relations on $9 \mathrm{q} 34.2$ (Figure 2). The present study has $91.3 \%$ power to detect SNPs that account for $2 \%$ of the ALP variance at $5 \times 10^{-5}$ significance level; however, it has only $48.6 \%$ power to detect SNPs at $5 \times 10^{-8}$ significance level, hence $5 \times 10^{-5}$ was set to be the cutoff point in SNP selection. Seven SNPs met the selection criteria (see Method). Among them, rs657152 on 9q34.2, which has been reported to be associated with ALP in European and Japanese population $[16,17]$, was replicated with a $P$ value of $5.88 \times 10^{-9}$ in our GWAS set. Therefore, we did not validate this SNP in the second stage. The other 6 SNPs were selected for further validation.

Three SNPs located within or near the $A B O$ locus showed significant association with serum ALP in validation population $\left(P=6.63 \times 10^{-16}, P=7.54 \times 10^{-44}\right.$ and $P=1.10 \times 10^{-20}$ for rs8176720, rs651007, and rs7025162, respectively) (Table 1). Using each SNP as a covariate, we performed conditional analysis on the remaining 2 SNPs for their association with serum ALP. The results revealed that rs651007 was associated with serum ALP levels $\left(P=1.84 \times 10^{-23}\right)$ independently of the other two SNPs, and it accounted for $2.15 \%$ of the total variance of serum ALP (Additional file 3: Table S2). A significant difference in ALP levels among different genetic-inferred ABO blood groups was observed in GWAS dataset $(\mathrm{ALP}=79.00 \mathrm{U} / \mathrm{L}, 80.86 \mathrm{U} / \mathrm{L}, 91.16 \mathrm{U} / \mathrm{L}$ and $92.82 \mathrm{U} / \mathrm{L}$ in individuals with blood group $\mathrm{A}, \mathrm{AB}, \mathrm{B}$ and $\left.\mathrm{O}, P=1.57 \times 10^{-18}\right)$, similar to previous reports $[18,19]$. To further explore whether the effect of the lead SNP rs651007 was driven by ABO blood group, we introduced $\mathrm{ABO}$ blood group into the multivariable linear regression model. As expected, the association between ALP levels and rs651007 dramatically reduced after ABO blood group adjustment. ( $\beta=-0.036, P=0.029$, data not shown). Other three loci that mapped on 2p23.1 (rs1383023, $P=0.905)$, 2p12.2 (rs7594727 in CNNM3, $P=0.501$ ) and $17 \mathrm{q} 21.32$ (rs7214920 close to RPRML, $P=0.912$ ) failed to be replicated (Table 1). The unbalanced distribution of the demographic characteristics in the discovery and replication samples in the present study might partly contribute to the lack of replication.

\section{Genetic heterogeneity on ALP levels among studies}

GWAS results of the present study were compared with previously published studies that conducted in populations 


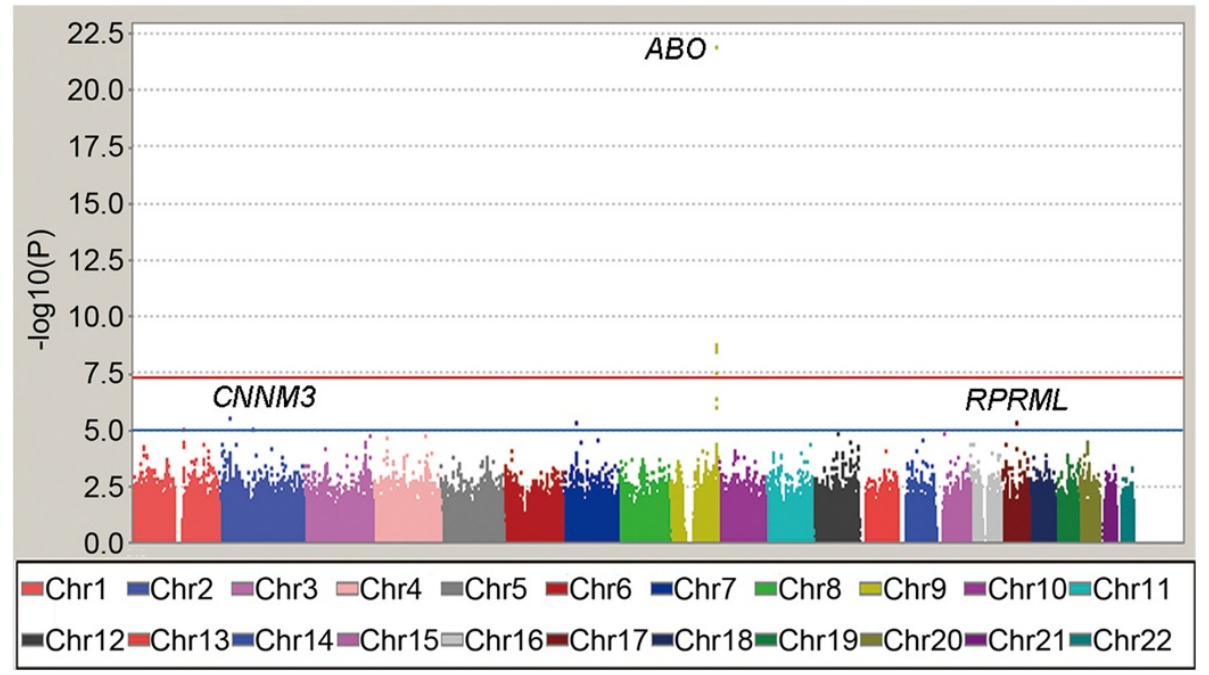

Figure 1 Manhattan plot of genome-wide association analyses for ALP. The horizontal axis shows the chromosomal positions while the vertical axis shows $-\log 10 P$ values from the test of association by linear regression analysis. The red horizontal line shows the $P$ value of $5.0 \times 10^{-8}$, and the blue horizontal line corresponds to a $P$ value of $1.0 \times 10^{-5}$.

of European and Japanese origins [15-17] (Table 2). Although $A B O$ locus found in other populations has been replicated in the present study, other loci identified in one or two earlier studies failed to be replicated. We did not observe positive signals on ALPL or GPLD1, which has been reported in European and Japanese studies (for ALPL, $P$ for lead SNP is $7.0 \times 10^{-15}, 5.08 \times 10^{-13}$ and in $3.70 \times 10^{-2}$ in European study, Japanese study and the present study, respectively; for GPLD1, $P$ for lead SNP is $1.2 \times 10^{-11}, 2.13 \times 10^{-11}$ and in $1.61 \times 10^{-3}$ in European study, Japanese study and the present study, respectively). Many loci identified in populations of European origin, e.g. ABCB11, PPP1R3B, C9orf125, REEP3, ST3GAL4 and $A B H D 12$, are neither significant in Japanese populations nor in our population although some of them got comparable effect sizes across studies (e.g. for rs12355784 on $J M J D 1 C, P=5.0 \times 10^{-10}, 8.66 \times 10^{-3}$ and $1.60 \times 10^{-2}$, and effect size $=0.025,0.049$ and 0.029 in European study, Japanese study and the present study, respectively). Only rs7173947, which mapped on an intergenic region on chromosome 15, has been identified in Japanese population with no significant signals around the locus being seen in European or in the present study. The heterogeneity test showed considerable heterogeneity across studies for some SNPs (e.g. for rs2242420 on $A L P L, P$ of $\mathrm{Q}=2.61 \times 10^{-6}$, $\left.\mathrm{I}^{2}=0.955\right)$, which may attribute to ethnic heterogeneity and differences in characteristics of samples and study designs, etc. The small sample size also limited the power of the present study to replicate SNPs with low MAF and/or minor effects (for SNPs that explain 1.0\% of the ALP variance with MAF of 0.05 , a sample size of 2,400 is needed to detect the association with $80 \%$ discovery power at $5 \times 10^{-5}$ significance level).

\section{Gene-environment interaction analysis}

Multiple factors have been proved to influence serum ALP levels, including age, gender, BMI, smoking, drinking and physical activity $[10-13,20]$. Therefore, we tested whether the independent SNP rs651007 interacted with age, gender, BMI (normal/overweight or obese), smoking or drinking on affecting ALP concentrations. As shown in Additional file 4: Table S3, rs651007 had a significant interaction with overweight and obesity on serum ALP levels $(F D R=0.036)$. For GG genotype carriers, the mean of serum ALP were similar between those with normal weight and those who were overweight or obese (89.52 U/L in overweight and obese subjects vs. $89.93 \mathrm{U} / \mathrm{L}$ in subjects with normal weight); GA or AA genotype carriers have significantly decreased serum ALP concentrations compared with GG genotype carriers; however, this effect was attenuated in overweight or obese individuals ( $\beta$ for rs651007 $=-0.091$ and -0.069 in subjects with normal weight and subjects who were overweight or obese, respectively). Thus, among AA genotype carriers, overweight or obese individuals had higher ALP levels than those with normal weight (81.24 U/L in overweight and obese subjects vs. $77.93 \mathrm{U} / \mathrm{L}$ in normal weight subjects) (Figure 3). The statistic power of interaction analysis in the present study is $71.1 \%$. No significant gene-environment interactions were observed between rs651007 and other factors including age, gender, drinking, and smoking (Additional file 4: Table S3).

\section{Discussion}

In the present two-stage GWAS, we confirmed that previously identified locus $A B O$ was associated with serum ALP levels in a Chinese population. When comparing the present study with previously published findings, ethnic 
Table 1 SNPs associated with serum ALP levels

\begin{tabular}{|c|c|c|c|c|c|c|c|c|c|c|c|c|c|c|}
\hline \multirow[t]{2}{*}{ SNP } & \multirow[t]{2}{*}{ Region } & \multirow[t]{2}{*}{ Position $^{\mathrm{a}}$} & \multirow[t]{2}{*}{ Genes } & \multirow[t]{2}{*}{ Locus } & \multirow{2}{*}{$\begin{array}{l}\text { Minor/major } \\
\text { allele }\end{array}$} & \multicolumn{3}{|c|}{ GWAS $^{\mathbf{b}}$} & \multicolumn{3}{|c|}{ Validation $^{\mathrm{b}^{*}}$} & \multicolumn{3}{|c|}{ Combined $^{\mathbf{b}}$} \\
\hline & & & & & & MAF & Effect size (s.e.m) & $P$ values & MAF & Effect size (s.e.m) & $P$ values & MAF & Effect size (s.e.m) & $P$ values \\
\hline rs1383023 & $2 p 23.1$ & 31872045 & $N / A$ & - & $\mathrm{A} / \mathrm{C}$ & 0.093 & $0.087(0.018)$ & $2.30 \times 10^{-6}$ & 0.075 & $0.0016(0.014)$ & $9.05 \times 10^{-1}$ & 0.080 & $-0.0009(0.005)$ & $8.59 \times 10^{-1}$ \\
\hline rs7594727 & $2 p 11.2$ & 96853597 & CNNM3 & Intron & $\mathrm{A} / \mathrm{C}$ & 0.107 & $-0.070(0.017)$ & $3.70 \times 10^{-5}$ & 0.116 & $-0.008(0.011)$ & $5.01 \times 10^{-1}$ & 0.113 & $-0.018(0.009)$ & $4.39 \times 10^{-2}$ \\
\hline rs8176720 & $9 q 34.2$ & 135122694 & $A B O$ & Exon & $C / T$ & 0.444 & $0.055(0.010)$ & $8.19 \times 10^{-8}$ & 0.454 & $0.038(0.005)$ & $6.63 \times 10^{-16}$ & 0.452 & $0.041(0.004)$ & $2.50 \times 10^{-21}$ \\
\hline rs651007 & $9 q 34.2$ & 135143696 & SURF6-ABO & Up- & $A / G$ & 0.244 & $-0.114(0.012)$ & $1.93 \times 10^{-21}$ & 0.222 & $-0.077(0.005)$ & $7.54 \times 10^{-44}$ & 0.226 & $-0.079(0.005)$ & $1.12 \times 10^{-56}$ \\
\hline rs7025162 & $9 q 34.2$ & 135156167 & SURF6-ABO & Up- & $A / G$ & 0.390 & $-0.064(0.011)$ & $2.20 \times 10^{-9}$ & 0.383 & $-0.044(0.005)$ & $1.10 \times 10^{-20}$ & 0.384 & $-0.047(0.004)$ & $2.82 \times 10^{-27}$ \\
\hline rs7214920 & $17 q 21.32$ & 42406128 & RPRML & Up- & $A / G$ & 0.119 & $0.068(0.016)$ & $2.55 \times 10^{-6}$ & 0.119 & $0.001(0.011)$ & $9.12 \times 10^{-1}$ & 0.119 & $0.020(0.009)$ & $2.99 \times 10^{-2}$ \\
\hline
\end{tabular}

${ }^{\mathrm{a}}$ Genome position is based on NCBI build 36.3 .

${ }^{\mathrm{b}}$ The $P$ values in GWAS stage are based on linear regression analysis on the log-transformed ALP with adjustment for age, gender and the top two eigenvectors in PCA analysis assuming an additive model by PLINK. The validation and combined $P$ values are calculated by linear regression model on the log-transformed ALP and adjusted for age and gender.

"SNPs of rs8176720, rs651007 and rs7025162 were validated in 8,830 healthy participants, while rs1383023, rs7594727 and rs7214920 were validated in 3,456 participants. 


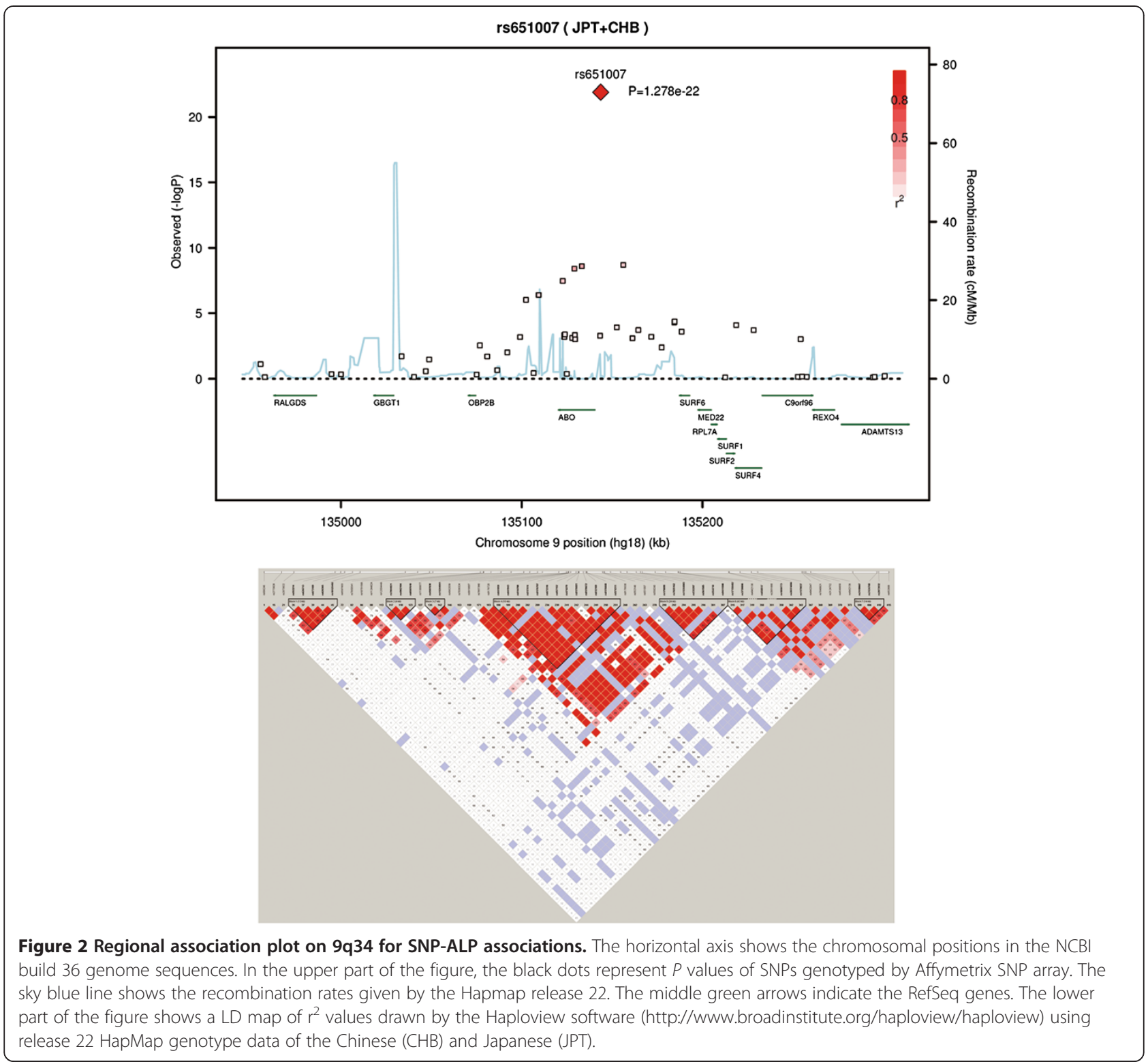

differences were observed across populations. In addition, a significant interaction between overweight and obesity and $A B O$ rs651007 on serum ALP was found. The effects of rs651007 on serum ALP levels were attenuated in overweight or obese individuals than in normal weight subjects.

The association between $A B O$ locus and serum ALP has been reported in other populations [15-17]. The present study was consistent with previous findings that serum ALP levels are much higher in individuals with blood type $\mathrm{B}$ or O [18]; the lead SNP rs651007 on $A B O$ locus, which is in high LD with previously reported $r s 495828\left(r^{2}=0.916\right)$, accounted for $2.15 \%$ of the total variance of serum ALP in our population, similar with that reported in earlier studies (2.0\% for rs657152 in Europeans and 3.79\% for rs495828 in Japanese) [16,17]. The coding product of human $A B O$ gene is a glycosyltransferase, of which catalytic activity could facilitate the transfer of carbohytrates to $\mathrm{H}$ antigen to form the antigenic structure of the $\mathrm{ABO}$ blood groups [21]. Current evidence found that human ABO blood groups or genetic variations on $A B O$ gene were associated with the risk of various diseases, such as pancreatic cancer, gastric cancer, falciparum malaria, venous thromboembolism, myocardial infarction and type 2 diabetes [22-28]. Genetic variations on $A B O$ gene were also found to be related with levels of serum E-selectin, sICAM, RBC, $\mathrm{Hb}$, vWF, and CEA $[16,25,29]$. In healthy fasted individuals, approximately $90 \%$ of serum ALP originate from liver/ bone/kidney, nearly $10 \%$ from intestine and in some cases $1 \%$ from placenta [5]. Most of the intestinal ALP is attached to $\mathrm{ABO}$ antigens on the surface of erythrocytes by a 
Table 2 Ethnic differences in major genetic variants associated with serum ALP levels

\begin{tabular}{|c|c|c|c|c|c|c|c|c|c|c|c|c|c|c|c|}
\hline \multirow[t]{2}{*}{ SNP } & \multirow[t]{2}{*}{ Gene } & \multirow[t]{2}{*}{ Chr } & \multirow{2}{*}{$\begin{array}{l}\text { Effect } \\
\text { allele }\end{array}$} & \multicolumn{3}{|c|}{ Present study (Chinese) ${ }^{a}$} & \multicolumn{3}{|c|}{ Japanese $^{b}$} & \multicolumn{3}{|c|}{$\mathrm{CEU}^{\mathrm{b}}$} & \multirow{2}{*}{$\begin{array}{c}P \text { value } \\
\text { of } Q\end{array}$} & \multirow[t]{2}{*}{$I^{2}$} & \multirow{2}{*}{$\begin{array}{l}\text { No. of } \\
\text { studies }\end{array}$} \\
\hline & & & & EAF & Effect size & $P$ value & EAF & Effect size & $P$ value & EAF & Effect size & $P$ value & & & \\
\hline rs1780324 & NBPF3-ALPL & 1 & $T$ & 0.29 & 0.020 & $9.56 \times 10^{-2}$ & 0.28 & 0.075 & $7.14 \times 10^{-5}$ & 0.44 & 0.031 & $7.0 \times 10^{-15}$ & 0.051 & 0.664 & 3 \\
\hline rs2242420 & ALPL & 1 & $\mathrm{~T}$ & 0.20 & -0.032 & $3.70 \times 10^{-2}$ & 0.18 & 0.163 & $5.08 \times 10^{-13}$ & 0.14 & - & - & $2.61 \times 10^{-6}$ & 0.955 & 2 \\
\hline rs16856332 & $A B C B 11$ & 2 & G & 0.06 & -0.026 & 0.270 & 0.07 & - & - & 0.39 & 0.031 & $1.6 \times 10^{-9}$ & 0.638 & 0.000 & 2 \\
\hline rs9467160 & GPLD1 & 6 & A & 0.03 & -0.032 & 0.369 & 0.003 & -0.05 & 0.76 & 0.21 & 0.034 & $1.2 \times 10^{-11}$ & 0.171 & 0.433 & 3 \\
\hline rs6911965 & GPLD1 & 6 & C & 0.11 & -0.056 & $1.61 \times 10^{-3}$ & 0.66 & -0.235 & $2.13 \times 10^{-11}$ & 0.17 & - & - & $5.35 \times 10^{-6}$ & 0.952 & 2 \\
\hline rs6984305 & PPPIR3B & 8 & A & 0.01 & 0.010 & 0.863 & 0 & - & - & 0.11 & 0.027 & $2.1 \times 10^{-10}$ & 0.772 & 0.000 & 2 \\
\hline rs2954021 & TRIB1 & 8 & A & 0.41 & 0.035 & $2.51 \times 10^{-3}$ & 0.43 & - & - & 0.50 & 0.014 & $2.3 \times 10^{-13}$ & 0.095 & 0.641 & 2 \\
\hline rs10819937 & C9orfi25 & 9 & C & 0.36 & 0.020 & 0.114 & 0.32 & - & - & 0.17 & 0.025 & $1.0 \times 10^{-9}$ & 0.741 & 0.000 & 2 \\
\hline rs657152 & $A B O$ & 9 & T & 0.46 & -0.062 & $3.78 \times 10^{-8}$ & 0.44 & -0.223 & $1.35 \times 10^{-38}$ & 0.38 & -0.047 & $1.7 \times 10^{-30}$ & 0 & 0.975 & 3 \\
\hline rs12355784 & JMJD1C & 10 & C & 0.33 & 0.029 & $1.60 \times 10^{-2}$ & 0.50 & 0.046 & $8.66 \times 10^{-3}$ & 0.49 & 0.025 & $5.0 \times 10^{-10}$ & 0.558 & 0.000 & 3 \\
\hline rs10761779 & REEP3 & 10 & G & 0.37 & 0.031 & $7.83 \times 10^{-3}$ & 0.45 & - & - & 0.49 & 0.025 & $6.9 \times 10^{-10}$ & 0.634 & 0.000 & 2 \\
\hline rs174601 & FADS2 & 11 & A & 0.41 & 0.007 & 0.563 & 0.38 & - & - & 0.35 & 0.017 & $2.6 \times 10^{-9}$ & 0.075 & 0.684 & 2 \\
\hline rs2236653 & ST3GAL4 & 11 & T & 0.70 & -0.024 & $4.40 \times 10^{-2}$ & 0.60 & - & - & 0.42 & 0.015 & $1.8 \times 10^{-9}$ & 0.488 & 0.000 & 2 \\
\hline rs7173947 & intergenic & 15 & C & 0.33 & -0.010 & 0.391 & 0.29 & -0.105 & $3.23 \times 10^{-8}$ & 0.41 & - & - & $2.75 \times 10^{-5}$ & 0.943 & 2 \\
\hline rs7186908 & PMFBP1 & 16 & C & 0.22 & -0.028 & $4.73 \times 10^{-2}$ & 0.34 & - & - & 0.24 & 0.02 & $4.8 \times 10^{-9}$ & $1.35 \times 10^{-3}$ & 0.903 & 2 \\
\hline rs314253 & DLG4 & 17 & G & 0.51 & 0.005 & 0.658 & 0.52 & - & - & 0.33 & 0.021 & $8.4 \times 10^{-12}$ & 0.179 & 0.447 & 2 \\
\hline rs281377 & FUT2 & 19 & T & 0.86 & 0.045 & $2.56 \times 10^{-2}$ & 0.87 & - & - & 0.43 & 0.018 & $1.1 \times 10^{-15}$ & $2.42 \times 10^{-3}$ & 0.891 & 2 \\
\hline rs7267979 & $A B H D 12$ & 20 & G & 0.07 & -0.031 & 0.173 & 0.10 & - & - & 0.57 & 0.015 & $7.4 \times 10^{-10}$ & 0.484 & 0.000 & 2 \\
\hline
\end{tabular}

a In order to get genotypes of all reported SNPs, imputed genotype data were used in the presented ethnic comparison table. EAF is short for effect allele frequency.

bQuoted from Chambers, et al. [15], Kamatani, et al. [16] and Yuan, et al. [17]. 


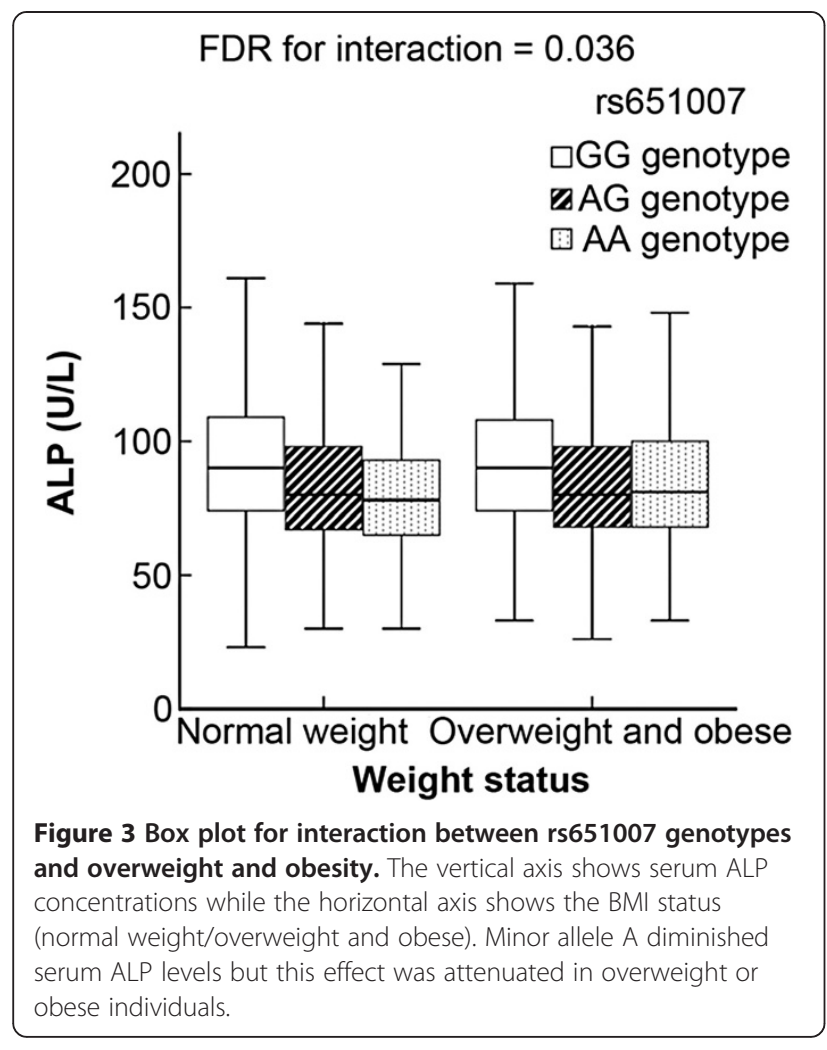

glycosyl-phosphatidylinositol anchor. Erythrocytes of blood type A bind to almost all intestinal ALP, while erythrocytes of blood type $\mathrm{B} / \mathrm{O}$ bind to a much lesser degree, therefore results in a more prevalent presence of intestinal ALP in serum in individuals with blood type $\mathrm{B} / \mathrm{O}[19,30]$. In this sense, the differences in the binding capacity between intestinal ALP and erythrocytes among individuals with different blood groups might be a potential mechanism underlying the association between ALP and ABO locus identified in GWAS. The observation that association between rs651007 and serum ALP dramatically reduced after $\mathrm{ABO}$ blood group adjustment also lend support to this deduction, indicating that $\mathrm{ABO}$ blood groups may act as the driving force behind this association.

Differences were observed among studies when comparing our findings with previously published results and parts of the reason might be due to the ethnic heterogeneity, such as varied effect allele frequencies and unique LD structures. For example, the MAF of rs16856332 are 0.39, 0.06 and 0.07 in Europeans, Chinese and Japanese, respectively; the MAF of rs 9467160 on GPLD1 locus is 0.21 in European but is only 0.03 in Chinese Han, and even lower, 0.003 in Japanese; the scenario is the same for rs7267979 on $A B H D 12$ that its effect allele frequency is 0.57 in European but is only 0.07 in Chinese. On the contrary, rs281377 has a MAF of 0.43 in European but the frequency of this effective allele is 0.86 in Chinese. Meanwhile, not all $A B O$ SNPs identified in Europeans are significant in Chinese, which may partially due to the differences in LD structure, e.g. the LD relation between rs 8176720 and rs514708 on $A B O$ gene is weak in Asian populations, but get stronger in Europeans $\left(\mathrm{r}^{2}=0.38,0.36\right.$ and 0.67 in the present, Japanese and European populations). Unique population structures, including different modifier genes, gene-gene or gene-environment interactions, different lifestyles or environment exposures can also be explanations for the heterogeneity across populations [31]. Notably, although it showed no evidence for heterogeneity across studies for SNPs on ABCB11, PPP1R3B, C9orf125, JMJD1C, REEP3, ST3GAL4, and ABHD12, SNPs on ALPL, GPLD1, $P M F B P 1$ and FUT2 represented considerable heterogeneity among studies. The lack of replication of many loci may also due to different sample selection criteria, different study designs and statistical analysis methods, as well as the relatively small sample size of the GWAS stage of the present study. Studies with larger sample size, different ethnic sources and multi-center cooperations are needed to explain the ethnic differences better.

Previous study found that serum ALP was higher in obese patients [32]. The interaction between rs651007 and overweight and obesity on ALP levels found in the present study may be one of the explanations. The effect of rs651007 on serum ALP was attenuated in overweight or obese individuals compared with normal weight subjects, resulting in higher ALP levels in overweight or obese individuals. eQTL analysis [33] showed that rs651007 could trans-regulate the expression of TNFRSF1A (Effect $=0.403$, $P=1.30 \times 10^{-5}$, LOD $=4.126$ ); meanwhile, rs651007 was observed associated to higher serum TNF-R2 levels in a previous study [25], demonstrating that rs651007 may be involved in the regulation of TNF receptors. In addition, TNF signaling is believed playing an important role in obesity since TNF $\alpha$ mRNA or protein was found overexpressed in obese subjects in both experimental and epidemiological studies. Researchers also found that TNF signaling plays an important role in the insulin resistance of obesity $[34,35]$. Moreover, TNF- $\alpha$ has been demostrated to positively regulate ALP levels in various types of cells [36-39]. Taken together, all evidence suggests that the interaction between rs651007 and overweight and obesity on serum ALP levels may act through the regulation of TNF system. Further studies are needed to uncover the real mechanism of the interaction.

Our study are not only consistent with findings of previous GWA studies [15-17], but also in line with early reports that serum ALP levels varied in individuals with different ABO blood types $[2,4,18]$, However, it is undeniable that the relatively small sample size of our GWAS stage limited us to detect SNPs with minor effects and SNPs with low MAF, which calls for further large sample size studies and consortium cooperation. 


\section{Conclusions}

In summary, our study confirmed that $A B O$ locus was a major determinant for serum ALP levels in Chinese Han population. When comparing the present study with previously published findings, ethnic differences were observed across populations. More importantly, we found that overweight and obesity could modify the effects of $A B O$ rs651007 polymorphism on serum ALP levels. Findings herein may furnish clinical application in prognosis control. Further studies are warranted to validate our findings and explore the potential mechanisms.

\section{Methods}

\section{Study subjects}

We performed a two-stage GWAS for serum ALP levels in individuals of Chinese Han origin, all of whom were recruited from Dongfeng-Tongji cohort study (DFTJ-cohort) in Hubei, China. DFTJ-cohort, which includes 27,009 retired employees from a state-owned automobile enterprise in China, was launched in 2008 and will be followed up every 5 years. Detailed information on demographics, lifestyle factors, baseline occupational and environmental exposures has been collected and a biospecimen bank (fasting blood serum, plasma, and DNA) is available as well. The main goal of the study was to identify environmental and genetic risk factors for chronic diseases, to investigate gene-environment interactions, and to find novel biomarkers for chronic diseases prediction [40].

The discovery set (GWAS stage) included 1461 cohort participants and the validation set (second stage) contained another 8830 cohort subjects. Participants who were deemed to be healthy in physical examinations were selected, and those who have self-reported severe diseases, such as stroke, coronary heart disease, cancers and diabetes mellitus, were excluded. The protocol was approved by ethics committee in Tongji Medical College and all subjects provided written informed consents.

\section{Alkaline phosphatase measurement}

Serum ALP levels were measured by ARCHITECT Ci8200 automatic analyzer (ABBOTT Laboratories. Abbott Park, Illinois, U.S.A) using Abbott Diagnostics reagents following standard experimental procedures from the manufacturer. This measurement was accomplished by the Biochemical Laboratory of Dongfeng Central Hospital, Shiyan, Hubei, China together with the quantification of other biochemical traits [40]. The intra-assay and inter-assay coefficients of variation for ALP were $5.35 \%$ and $3.25 \%$.

\section{SNP genotyping and quality control}

In discovery stage, a genome-wide genotyping scan in 1461 subjects was carried out using Affymetrix Genome-Wide Human SNP Array 6.0 chips. High-quality genotyping was performed by commercial company specialized in
Affymetrix SNP array genotyping following standard experimental procedures from the manufacturer. A total of 906,703 SNPs were genotyped among 1,461 subjects in which 38,446 SNPs not mapped on autosomes were excluded. After QC filtering, SNPs with MAF $<0.01$ (193,732 SNPs), HWE < 0.0001 (1,332 SNPs), and SNPs call rate $<95 \%(17,764$ SNPs) were excluded. Individuals with a call rate $<95 \%$ were also ruled out for further analysis. Finally, 1,452 subjects with 658,288 autosomal SNPs were retained for statistical analyses, with an overall call rate of 99.68\%. In validation stage, SNPs were genotyped using the iPLEX system (Sequenom, Inc., San Diego, CA, USA), in which all genotyping reactions were performed in 384-well plates according to the manufacturer's iPLEX Application Guide (Sequenom, Inc.). Each plate included four randomly selected duplicates, as well as six negative controls using double distilled water. The average concordance rate for genotyping in validation was $99.8 \%$.

\section{Statistical analysis}

The population structure was evaluated by PCA using the software package EIGENSTRAT 3.0 [41], and GWAS data was analyzed utilizing PLINK $1.06[42,43]$. Quantile-quantile plot was plotted by R 2.11.1 [44], Manhattan plot of - $\log 10$ $P$ was generated with Haploview (v4.1) [45], and the regional association plot was generated using SNAP [46]. To infer ungenotyped SNPs [47], MACH 1.0 software [48] was applied to impute ungenotyped SNPs using LD information from the HapMap phase II database (CHB + JPT as a reference set (2007-08_rel22, released 2007-03-02)) [49]. Imputed SNPs with high genotype information content (Rsq $>0.3$ ) were kept for the further association analysis, which was conducted using ProbABEL software [50,51] Quanto 1.2.4 was used for power calculation [52]. BMI was calculated as the individual's body mass $(\mathrm{kg})$ divided by the square of his or her height $\left(\mathrm{m}^{2}\right)$. Individuals whose BMI was greater than or equal to $24 \mathrm{~kg} / \mathrm{m}^{2}$ were defined as overweight and obese, otherwise were classed as normal weight [53-55]. Individuals who had smoked at least one cigarette per day for more than half a year no matter currently or formerly were defined as smokers, otherwise were defined as non-smokers. Those who had drunk at least once a week for more than half a year no matter currently or formerly were classed as drinkers, else were classed as non-drinkers. Prior to analyses, serum ALP level was nature log-transformed to normalize the distribution. For GWA analysis, ALP-SNP associations were tested assuming an additive genetic model using linear regression with age, gender and the top two eigenvectors in PCA being included as covariates. SNPs that met the following criteria were selected for further validation: (1) SNPs with $P \leq 5.0 \times 10^{-5}$ in GWAS stage; (2) when multiple SNPs showed strong LD $\left(\mathrm{r}^{2} \geq 0.8\right)$, SNP with the lowest $P$ value was selected and (3) $\mathrm{MAF} \geq 0.05$. In order to extract genotype information for 
all reported SNPs from the present study, imputed genotype data was used for ethnic comparisons. Loci that were positive $\left(P<5.0 \times 10^{-8}\right.$ in GWAS stage $)$ in either previously published studies or the present study were gathered, and representative SNPs on each locus were selected and compared. GWAMA [56] was applied to calculate heterogeneity among studies with the option of random effect correction being abled.

SNP that showed a $P<0.05 / 6$ in the validation dataset or a $P<5 \times 10^{-8}$ in the combined dataset is considered to be statistically significant. For gene-environment interaction analysis, we fitted linear regression models by adding an interaction term of SNPs and environmental factors: $\mathrm{Y}=\alpha+\beta \mathrm{s}^{*} \mathrm{SNP}+\beta \mathrm{se} \mathrm{s}^{*} \mathrm{SNP} *$ environment $+\beta \mathrm{e}^{*}$ environment + $\beta c^{*} C$; where SNP represented SNP to be tested (in the present situation, rs651007); environmental factors included age, gender, BMI (normal/overweight and obese), smoking and drinking, which were introduced into the model separately; and $\mathrm{C}$ represented covariates including age and gender. We applied the false discovery rate (FDR) approach [57] to control for multiple comparisons (1 SNP $\times 5$ environmental factors $=5$ times $)$ in the interaction analysis. Using each of the SNPs as a covariate, we performed conditional analysis on the remaining 2 SNPs on $A B O$ gene for their association with ALP. The proportion of the total serum ALP variation explained by each SNP was measured by $r^{2}$, which is the difference of the model sum of squares between models with and without the SNP of interest divided by the corrected total sum of squares of the full model. Two tagging SNPs (rs505922 and rs8176746) in $A B O$ gene were used to infer ABO blood group for each individual in GWAS dataset. $\mathrm{ABO}$ blood group was set into a variable of integer according to the reported binding capacity between $A B O$ antigen and $\mathrm{ALP}(\mathrm{A}=0, \mathrm{AB}=1, \mathrm{~B}=2, \mathrm{O}=3)[19,30,58]$. These analyses were performed using SAS version 9.2 (SAS Institute, Cary, NC).

\section{Additional files}

Additional file 1: Table S1. Clinical characteristics of the participants in the discovery and validation datasets from DFTJ-cohort Study.

Additional file 2: Figure S1. Q-Q plots of GWAS for ALP in discovery set. Description of data: The horizontal axis shows -log10 transformed expected $P$ values, while the vertical axis indicates - log 10 transformed observed $P$ values. The genomic inflation factor $\lambda$ is 1.011 .

Additional file 3: Table S2. Variance of serum ALP level explained by SNPS. Additional file 4: Table S3. Interactions between ABO rs651007 and drinking, smoking, gender and overweight and obesity.

\section{Competing interests}

The authors declare that they have no competing interests.

\section{Authors' contributions}

$J$ participated in the genotyping, performed the statistical analysis and drafted the manuscript. $\mathrm{MH}$ contributed to the conception and design, involved in drafting the manuscript and revising it critically for important intellectual content. LG and YH performed the statistical analysis. CW, LZ, HG contributed to the acquisition of data and SNPS selection. JY, JZ, XM, ML, DL, $\mathrm{HY}, \mathrm{FBH}, \mathrm{DL}$ and TW have been involved in acquisition of data and contributed to conception and design. BY, XD, QD, SH, LG, DH, SD and TW have been involved in acquisition of data. All authors read and approved the final manuscript.

\section{Acknowledgments}

The authors would like to thank all volunteers for assisting in clinic data, questionnaire data and samples collection. The authors appreciate the financial support provided by the Program of Introducing Talents of Discipline to Universities to T.W., the Program for New Century Excellent Talents (NCET) and the National Natural Science Foundation of China (81172751) to M.H

\section{Author details}

${ }^{1}$ MOE Key Lab of Environment and Health, School of Public Health, Tongji Medical College, Huazhong University of Science \& Technology, 430030 Wuhan, Hubei, China. ${ }^{2}$ State Key Laboratory of Molecular Oncology, Cancer Institute \& Hospital, Chinese Academy of Medical Sciences and Peking Union Medical College, 100021 Beijing, China. ${ }^{3}$ Dongfeng Central Hospital, Dongfeng Motor Corporation and Hubei University of Medicine, 442008 Shiyan, Hubei, China. ${ }^{4}$ Departments of Nutrition and Epidemiology, Harvard School of Public Health, Boston, MA 02115, USA. ${ }^{5}$ Department of Epidemiology, School of Public Health and Management, Chongqing Medical University, 400016 Chongqing, China.

Received: 22 November 2012 Accepted: 24 September 2013 Published: 5 October 2013

\section{References}

1. Schoppet M, Shanahan CM: Role for alkaline phosphatase as an inducer of vascular calcification in renal failure? Kidney Int 2008, 73:989-991.

2. Kaplan MM: Alkaline phosphatase. N Engl J Med 1972, 286:200-202.

3. Moss DW: Perspectives in alkaline phosphatase research. Clin Chem 1992, 38:2486-2492.

4. Cho SR, Lim YA, Lee WG: Unusually high alkaline phosphatase due to intestinal isoenzyme in a healthy adult. Clin Chem Lab Med 2005, 43:1274-1275.

5. Hirano K, Matsumoto H, Tanaka T, Hayashi Y, lino S, Domar U, Stigbrand T: Specific assays for human alkaline phosphatase isozymes. Clin Chim Acta 1987, 166:265-273.

6. Korostishevsky M, Cohen Z, Malkin I, Ermakov S, Yarenchuk O, Livshits G: Morphological and biochemical features of obesity are associated with mineralization genes' polymorphisms. Int J Obes 2010, 34:1308-1318.

7. Blayney MJ, Pisoni RL, Bragg-Gresham JL, Bommer J, Piera L, Saito A, Akiba $T$, Keen ML, Young EW, Port FK: High alkaline phosphatase levels in hemodialysis patients are associated with higher risk of hospitalization and death. Kidney Int 2008, 74:655-663.

8. Regidor DL, Kovesdy CP, Mehrotra R, Rambod M, Jing J, McAllister CJ, Van Wyck D, Kopple JD, Kalantar-Zadeh K: Serum alkaline phosphatase predicts mortality among maintenance hemodialysis patients. J Am Soc Nephrol 2008, 19:2193-2203.

9. Tonelli M, Curhan G, Pfeffer M, Sacks F, Thadhani R, Melamed ML, Wiebe N, Muntner $P$ : Relation between alkaline phosphatase, serum phosphate, and all-cause or cardiovascular mortality. Circulation 2009, 120:1784-1792.

10. Wannamethee SG, Shaper AG: Cigarette smoking and serum liver enzymes: the role of alcohol and inflammation. Ann Clin Biochem 2010, 47:321-326

11. Gordon T: Factors associated with serum alkaline phosphatase level. Arch Pathol Lab Med 1993, 117:187-190.

12. Lester ME, Urso ML, Evans RK, Pierce JR, Spiering BA, Maresh CM, Hatfield DL, Kraemer WJ, Nindl BC: Influence of exercise mode and osteogenic index on bone biomarker responses during short-term physical training. Bone 2009, 45:768-776.

13. Clark LC Jr, Beck E: Plasma alkaline phosphatase activity; normative data for growing children. J Pediatr 1950, 36:335-341.

14. Srivastava AK, Masinde G, Yu H, Baylink DJ, Mohan S: Mapping quantitative trait loci that influence blood levels of alkaline phosphatase in MRL/MpJ and SJL/J mice. Bone 2004, 35:1086-1094. 
15. Chambers JC, Zhang W, Sehmi J, Li X, Wass MN, Van der Harst P, Holm H, Sanna S, Kavousi M, Baumeister SE, et al: Genome-wide association study identifies loci influencing concentrations of liver enzymes in plasma. Nat Genet 2011, 43:1131-1138.

16. Kamatani $Y$, Matsuda K, Okada Y, Kubo M, Hosono N, Daigo Y, Nakamura Y, Kamatani N: Genome-wide association study of hematological and biochemical traits in a Japanese population. Nat Genet 2010, 42:210-215

17. Yuan X, Waterworth D, Perry JR, Lim N, Song K, Chambers JC, Zhang W, Vollenweider P, Stirnadel H, Johnson T, et al: Population-based genome-wide association studies reveal six loci influencing plasma levels of liver enzymes. Am J Hum Genet 2008, 83:520-528.

18. Bamford KF, Harris H, Luffman JE, Robson EB, Cleghorn TE: Serum-AlkalinePhosphatase and the Abo Blood-Groups. Lancet 1965, 1:530-531.

19. Bayer PM, Hotschek $H$, Knoth E: Intestinal alkaline phosphatase and the ABO blood group system-a new aspect. Clin Chim Acta 1980, 108:81-87.

20. Frost-Pineda K, Liang Q, Liu J, Rimmer L, Jin Y, Feng S, Kapur S, Mendes P, Roethig H, Sarkar M: Biomarkers of potential harm among adult smokers and nonsmokers in the total exposure study. Nicotine Tob Res 2011, 13:182-193.

21. Greenwell P: Blood group antigens: molecules seeking a function? Glycoconj J 1997, 14:159-173.

22. Nakao M, Matsuo K, Hosono S, Ogata S, Ito H, Watanabe M, Mizuno N, lida S, Sato $S$, Yatabe $Y$, et al: ABO blood group alleles and the risk of pancreatic cancer in a Japanese population. Cancer Sci 2011, 102:1076-1080.

23. Nakao M, Matsuo K, Ito H, Shitara K, Hosono S, Watanabe M, Ito S, Sawaki A, lida $S$, Sato $S$, et al: ABO genotype and the risk of gastric cancer, atrophic gastritis, and Helicobacter pylori infection. Cancer Epidemiol Biomarkers Prev 2011, 20:1665-1672

24. Panda AK, Panda SK, Sahu AN, Tripathy R, Ravindran B, Das BK: Association of $A B O$ blood group with severe falciparum malaria in adults: case control study and meta-analysis. Malar J 2011, 10:309.

25. Qi L, Cornelis MC, Kraft P, Jensen M, Van Dam RM, Sun Q, Girman CJ, Laurie CC, Mirel DB, Hunter DJ, et al: Genetic variants in $A B O$ blood group region, plasma soluble E-selectin levels and risk of type 2 diabetes. Hum Mol Genet 2010, 19:1856-1862.

26. Reilly MP, Li M, He J, Ferguson JF, Stylianou IM, Mehta NN, Burnett MS, Devaney JM, Knouff CW, Thompson JR, et al: Identification of ADAMTS7 as a novel locus for coronary atherosclerosis and association of $A B O$ with myocardial infarction in the presence of coronary atherosclerosis: two genome-wide association studies. Lancet 2011, 377:383-392.

27. Wolpin BM, Kraft P, Gross M, Helzlsouer K, Bueno-de-Mesquita HB, Steplowski E, Stolzenberg-Solomon RZ, Arslan AA, Jacobs EJ, Lacroix A, et al: Pancreatic cancer risk and $A B O$ blood group alleles: results from the pancreatic cancer cohort consortium. Cancer Res 2010, 70:1015-1023.

28. Wu O, Bayoumi N, Vickers MA, Clark P: $A B O(H)$ blood groups and vascular disease: a systematic review and meta-analysis. J Thromb Haemost 2008, 6:62-69.

29. Tregouet DA, Heath S, Saut N, Biron-Andreani C, Schved JF, Pernod G, Galan $P$, Drouet $L$, Zelenika D, Juhan-Vague I, et al: Common susceptibility alleles are unlikely to contribute as strongly as the FV and ABO loci to VTE risk: results from a GWAS approach. Blood 2009, 113:5298-5303.

30. Deng JT, Hoylaerts MF, Van Hoof VO, De Broe ME: Differential release of human intestinal alkaline phosphatase in duodenal fluid and serum. Clin Chem 1992, 38:2532-2538.

31. Wang F, Xu CQ, He Q, Cai JP, Li XC, Wang D, Xiong X, Liao YH, Zeng QT, Yang YZ, et al: Genome-wide association identifies a susceptibility locus for coronary artery disease in the Chinese Han population. Nat Genet 2011, 43:345-349.

32. Andersen T, Christoffersen P, Gluud C: The liver in consecutive patients with morbid obesity: a clinical, morphological, and biochemical study. Int J Obes 1984, 8:107-115.

33. Dixon AL, Liang L, Moffatt MF, Chen W, Heath S, Wong KC, Taylor J, Burnett E, Gut I, Farrall M, et al: A genome-wide association study of global gene expression. Nat Genet 2007, 39:1202-1207.

34. Aldhahi W, Hamdy O: Adipokines, inflammation, and the endothelium in diabetes. Curr Diab Rep 2003, 3:293-298.

35. Olszanecka-Glinianowicz M, Zahorska-Markiewicz B, Janowska J, Zurakowski A: Serum concentrations of nitric oxide, tumor necrosis factor (TNF)-alpha and TNF soluble receptors in women with overweight and obesity. Metabolism 2004, 53:1268-1273.

36. Shioi A, Katagi M, Okuno Y, Mori K, Jono S, Koyama H, Nishizawa Y: Induction of bone-type alkaline phosphatase in human vascular smooth muscle cells: roles of tumor necrosis factor-alpha and oncostatin M derived from macrophages. Circ Res 2002, 91:9-16.

37. Ding J, Ghali O, Lencel P, Broux O, Chauveau C, Devedjian JC, Hardouin P, Magne D: TNF-alpha and IL-1beta inhibit RUNX2 and collagen expression but increase alkaline phosphatase activity and mineralization in human mesenchymal stem cells. Life Sci 2009, 84:499-504.

38. Lencel P, Delplace S, Hardouin P, Magne D: TNF-alpha stimulates alkaline phosphatase and mineralization through PPARgamma inhibition in human osteoblasts. Bone 2011, 48:242-249.

39. Tintut $Y$, Patel J, Parhami F, Demer LL: Tumor necrosis factor-alpha promotes in vitro calcification of vascular cells via the cAMP pathway. Circulation 2000, 102:2636-2642.

40. Wang F, Zhu J, Yao P, Li X, He M, Liu Y, Yuan J, Chen W, Zhou L, Min X, et al: Cohort profile: The Dongfeng-Tongji cohort study of retired workers. Int J Epidemiol 2013, 42:731-740.

41. Price AL, Patterson NJ, Plenge RM, Weinblatt ME, Shadick NA, Reich D: Principal components analysis corrects for stratification in genome-wide association studies. Nat Genet 2006, 38:904-909.

42. PLINK. http://pngu.mgh.harvard.edu/ purcell/plink/.

43. Purcell S, Neale B, Todd-Brown K, Thomas L, Ferreira MA, Bender D, Maller J, Sklar P, De Bakker PI, Daly MJ, Sham PC: PLINK: a tool set for whole-genome association and population-based linkage analyses. Am J Hum Genet 2007, 81:559-575.

44. R statistical environment. http://cran.r-project.org/.

45. Barrett JC, Fry B, Maller J, Daly MJ: Haploview: analysis and visualization of LD and haplotype maps. Bioinformatics 2005, 21:263-265.

46. Johnson AD, Handsaker RE, Pulit SL, Nizzari MM, O'Donnell CJ, De Bakker PI: SNAP: a web-based tool for identification and annotation of proxy SNPs using HapMap. Bioinformatics 2008, 24:2938-2939.

47. Marchini J, Howie B, Myers S, McVean G, Donnelly P: A new multipoint method for genome-wide association studies by imputation of genotypes. Nat Genet 2007, 39:906-913.

48. MACH 1.0. http://www.sph.umich.edu/csg/abecasis/mach/

49. International HapMap Project. http://www.hapmap.org/index.html.

50. Aulchenko YS, Struchalin MV, Van Duijn CM: ProbABEL package for genome-wide association analysis of imputed data. BMC Bioinformatics 2010, 11:134.

51. Cochran WG: The Combination of Estimates from Different Experiments. Biometrics 1954, 10:101-129.

52. Quanto. http://hydra.usc.edu/gxe/

53. Zhou BF: Effect of body mass index on all-cause mortality and incidence of cardiovascular diseases-report for meta-analysis of prospective studies open optimal cut-off points of body mass index in Chinese adults. Biomed Environ Sci 2002, 15:245-252.

54. Wu Y: Overweight and obesity in China. BMJ 2006, 333:362-363.

55. Zhou BF: Cooperative Meta-Analysis Group of the Working Group on Obesity in C: Predictive values of body mass index and waist circumference for risk factors of certain related diseases in Chinese adults-study on optimal cut-off points of body mass index and waist circumference in Chinese adults. Biomed Environ Sci 2002, 15:83-96.

56. GWAMA. http://www.well.ox.ac.uk/gwama/index.shtml.

57. Benjamini $Y$, Hochberg $Y$ : Controlling the false discovery rate: a practical and powerful approach to multiple testing. J Roy Stat Soc 1995, 57:289-300.

58. Tanikawa C, Urabe Y, Matsuo K, Kubo M, Takahashi A, Ito H, Tajima K, Kamatani N, Nakamura Y, Matsuda K: A genome-wide association study identifies two susceptibility loci for duodenal ulcer in the Japanese population. Nat Genet 2012, 44:430-434-S431-432.

doi:10.1186/1471-2164-14-684

Cite this article as: Li et al:: Genome-wide association study on serum alkaline phosphatase levels in a Chinese population. BMC Genomics 2013 14:684. 